\title{
Regularization of piecewise smooth singularly perturbed systems
}

\section{Pedro Toniol Cardin ${ }^{1}$}

Departamento de Matemática, FEIS-UNESP, Ilha Solteira, SP

Jaime Rezende de Moraes ${ }^{2}$

Departamento de Matemática, UEMS, Dourados, MS

Paulo Ricardo da Silva ${ }^{3}$

Departamento de Matemática, IBILCE-UNESP, São José do Rio Preto, SP

\begin{abstract}
In this work we deal with piecewise smooth singularly perturbed systems$$
\dot{x}=\left\{\begin{array}{ll}
F(x, y, \varepsilon) & \text { if } \quad h(x, y, \varepsilon) \leq 0, \\
G(x, y, \varepsilon) & \text { if } \quad h(x, y, \varepsilon) \geq 0,
\end{array} \quad \varepsilon \dot{y}=H(x, y, \varepsilon) .\right.
$$

In system (1), $\varepsilon \in \mathbb{R}$ is a non-negative small parameter, $x \in \mathbb{R}^{n}$ and $y \in \mathbb{R}$ denote the slow and fast variables, respectively, and $F, G, h$ and $H$ are $C^{r}$ maps which vary differentially with respect to $\varepsilon$, with $r \geq 1$. We study the Sotomayor-Teixeira regularization of periodic orbits of system (1) with $\varepsilon=0$ and with $\varepsilon>0$ sufficiently small. More specifically, we establish the persistence of periodic orbits with sewing or with sliding of system (1) with $\varepsilon=0$ and with $\varepsilon>0$ for their respective regularized systems.
\end{abstract}

Keywords. Singular perturbation, Filippov systems, regularization.

\section{Introduction}

Many systems of relevance to applications are modeled using piecewise smooth dynamical systems. The study of such systems has, in recent years, established an important frontier between Mathematics, Physics and Engineering. They appear in various situations like mechanical systems with dry friction or with impacts, in control theory, electronic, economics, medicine and biology. See [4] for a general scope of the matter.

In our approach Filippov convention [6] is considered. Filippov systems are systems modeled by different smooth ODEs (ordinary differential equations) in different open domains separated by smooth discontinuity boundaries. In the simplest case the phase space

\footnotetext{
${ }^{1}$ pedrocardin@mat.feis.unesp.br

2 jrezmoraes@gmail.com

${ }^{3}$ prs@ibilce.unesp.br
} 
is composed by two domains such that for each domain a different ODE governs the dynamics, namely

$$
\dot{x}=Z(x)= \begin{cases}F(x), & \text { if } \quad h(x) \leq 0, \\ G(x), & \text { if } h(x) \geq 0 .\end{cases}
$$

In equation (2), $F$ and $G$ are $C^{r}$ vector fields defined on the open set $U \subset \mathbb{R}^{n}$, with $r \geq 1$, and $h: U \rightarrow \mathbb{R}$ is a smooth function having 0 as a regular value. The common boundary $\Sigma=\{h(x)=0\}$ between the domains $\Sigma^{-}=\{h(x) \leq 0\}$ and $\Sigma^{+}=\{h(x) \geq 0\}$ is called switching manifold.

An important question with regards to investigations of the dynamics of systems (2) is the effect of singular perturbations. In this work we investigate how the dynamics of piecewise smooth systems is affected by singular perturbation. For that let us consider slow-fast systems of the form (1). It is worth to say that some papers have contributed to the study of this issue, see for instance the recent articles [1-3] and [7].

In this work we study the Sotomayor-Teixeira regularization of periodic orbits of system (1) with $\varepsilon=0$ and with $\varepsilon>0$ sufficiently small. We show that periodic orbits with sewing or with sliding of system (1) with $\varepsilon=0$ and with $\varepsilon>0$ are persistent for their respective regularized systems.

\section{Preliminaries}

In this section we present some basic facts about Filippov systems, the regularization process and piecewise smooth singularly perturbed systems.

\section{$2.1 \quad$ Filippov Systems}

We use the notation $F h(p)=F(p) \cdot \nabla h(p)$ for the scalar product in $\mathbb{R}^{n}$. For $k \geq 2$, we define inductively $F^{k} h(p)=F\left(F^{k-1} h\right)(p)$. On the switching manifold $\Sigma$ of system (2) the following open sets are distinguished: The sewing region $\Sigma^{c}=\{p \in \Sigma:[F h(p)][G h(p)]>$ $0\}$; the escaping region $\Sigma^{e}=\{p \in \Sigma: F h(p)<0, G h(p)>0\}$ and the sliding region $\Sigma^{s}=\{p \in \Sigma: F h(p)>0, G h(p)<0\}$. If a point of the phase space which is moving in an orbit of $Z=(F, G)$ falls onto $\Sigma^{c}$ then it crosses $\Sigma^{c}$ over to another part of the space. In $\Sigma^{e}$ and $\Sigma^{s}$, the definition of the local orbit is given by the Filippov convention [6]. We consider the vector field $Z^{\Sigma}$ which is the linear convex combination of $F$ and $G$ tangent to $\Sigma$, that is

$$
\dot{x}=Z^{\Sigma}(x)=\frac{F h(x) G(x)-G h(x) F(x)}{(F-G) h(x)} .
$$

We call $Z^{\Sigma}$ the sliding vector field associated to the Filippov system (2), independently whether it is defined in the sliding or escaping region. See Figure 1. Solutions of $Z=(F, G)$ through points of $\Sigma^{e} \cup \Sigma^{s}$ follow the orbit of $Z^{\Sigma}$.

Tangency points are points where one of the two vector fields $F$ or $G$ is tangent to $\Sigma$. They are characterized by $p \in \Sigma$ such that $F h(p)=0$ or $G h(p)=0$. Tangency points include the case $F(p)=0$ or $G(p)=0$, that is, when one of the two vector fields has an 
equilibrium point at $\Sigma$. We say that $p \in \Sigma$ is a regular point if $p \in \Sigma^{c}$ or $p \in \Sigma^{s} \cup \Sigma^{e}$ and $Z^{\Sigma}(p) \neq 0$. The points of $\Sigma$ which are not regular are called singular.

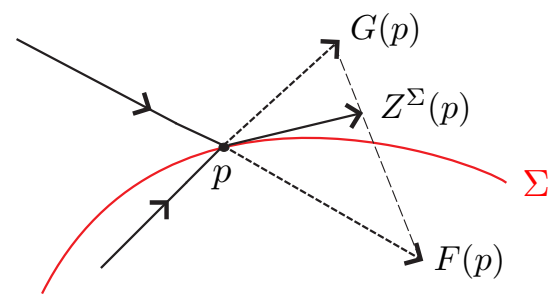

Figura 1: The sliding vector field $Z^{\Sigma}$.

\subsection{The regularization process}

The regularization process was introduced by Sotomayor and Teixeira in [9]. It is based in replacing the two adjacent vector fields by a $\lambda$-parametric vector field built as a linear convex combination of them in a $\lambda$-neighborhood of the switching manifold. More specifically, consider the non-smooth system (2). The regularization consists in an 1-parameter family of smooth vector fields $Z_{\lambda}$ on $U \subseteq \mathbb{R}^{n}$ such that for each $\lambda_{0}>0$,

(i) $Z_{\lambda_{0}}$ is equal to $G$ in all points of $\Sigma^{+}$whose distance to $\Sigma$ is bigger than $\lambda_{0}$.

(ii) $Z_{\lambda_{0}}$ is equal to $F$ in all points of $\Sigma^{-}$whose distance to $\Sigma$ is bigger than $\lambda_{0}$.

A $C^{\infty}$ function $\varphi: \mathbb{R} \rightarrow \mathbb{R}$ is a transition function if $\varphi(s)=-1$ for $s \leq-1, \varphi(0)=0$, $\varphi(s)=1$ for $s \geq 1$ and $\varphi^{\prime}(s)>0$ for $s \in(-1,1)$.

The regularization of system (2) is the one-parameter $\lambda$ family given by

$$
\dot{x}=Z_{\lambda}(x)=\frac{G(x)+F(x)}{2}+\varphi\left(\frac{h(x)}{\lambda}\right) \frac{G(x)-F(x)}{2},
$$

where $\varphi$ is a transition function. Denote $\varphi_{\lambda}=\varphi(h(x) / \lambda)$. The regularization zone is the region around the switching manifold $\Sigma$ given by $\varphi_{\lambda}^{-1}(-1,1)$.

\subsection{Piecewise smooth slow-fast systems}

For each $\varepsilon \geq 0$ we denote by $\Sigma_{\varepsilon}$ the switching manifold of (1), i.e. $\Sigma_{\varepsilon}=\{h(x, y, \varepsilon)=0\}$. The set $\mathcal{S}_{0}:=\{H(x, y, 0)=0\}$ is named critical manifold of the slow-fast system (1). Here we are supposing that $\Sigma_{0}$ and $\mathcal{S}_{0}$ are in general position. We will assume that $H(x, y, \varepsilon)=0$ can be solved by $y=f_{\varepsilon}(x)$, for all $\varepsilon \geq 0$. For $\varepsilon=0$ in (1) we have the reduced problem

$$
\dot{x}=\left\{\begin{array}{ll}
\tilde{F}(x) & \text { if } \quad \tilde{h}(x) \leq 0, \\
\tilde{G}(x) & \text { if } \quad \tilde{h}(x) \geq 0,
\end{array} \quad 0=H(x, y, 0),\right.
$$

where $\tilde{F}(x)=F\left(x, f_{0}(x), 0\right), \tilde{G}(x)=G\left(x, f_{0}(x), 0\right)$ and $\tilde{h}(x)=h\left(x, f_{0}(x), 0\right)$. The reduced problem $(5)$ is a dynamical system defined on the manifold $\mathcal{S}_{0}$. We will use the notations $\Sigma_{\varepsilon}^{c}, \Sigma_{\varepsilon}^{e}$ and $\Sigma_{\varepsilon}^{s}$ to denote the sewing, escaping and sliding regions associated to the Filippov slow-fast system (1), respectively, for all $\varepsilon \geq 0$. 
Definição 2.1. Let $K \subset \mathcal{S}_{0}$ be a compact set. We say that $K$ is normally hyperbolic if $H_{y}(x, y, 0)$ is nonzero, for all $(x, y) \in K$.

The following definition was given in [8].

Definição 2.2. Let $\Gamma$ be a periodic orbit with sewing or with sliding of system (5). We say that $\Gamma$ is hyperbolic if

- $\Gamma$ is a periodic orbit with sewing and $\eta^{\prime}(p) \neq 1$ where $\eta$ is the first return map defined on a segment $N$ with $p \in N \pitchfork \Gamma$;

- $\Gamma$ is a periodic orbit with sliding and all sliding arcs of $\Gamma$ are either sliding or escaping.

\section{Statement of the main result}

In what follows we define the regularization of systems (1) and (5). The regularized systems of (1) and (5) are given respectively by

$$
\begin{array}{ll}
\dot{x}=\frac{G+F}{2}+\varphi\left(\frac{h}{\lambda}\right) \frac{G-F}{2}, & \varepsilon \dot{y}=H, \\
\dot{x}=\frac{\widetilde{G}+\widetilde{F}}{2}+\varphi\left(\frac{\tilde{h}}{\lambda}\right) \frac{\widetilde{G}-\widetilde{F}}{2}, \quad H=0,
\end{array}
$$

where $\varphi$ is a transition function and all the functions in (6) are evaluated at $(x, y, \varepsilon)$ and all the function in $(7)$ are evaluated at $\left(x, f_{0}(x), 0\right)$.

We state below a result that ensures the persistence of hyperbolic periodic orbits with sewing or with sliding of the reduced problem (5) for the regularized systems (6) and (7).

Teorema 3.1. Consider a $C^{r}$ family like (1), with $x \in \mathbb{R}^{2}$ and $r \geq 1$. Let $\Gamma \subset \mathcal{S}_{0}$ be a hyperbolic periodic orbit with sewing or with sliding of the reduced problem (5). Then:

(a) For each small $\lambda>0$ the regularized system (7) has a hyperbolic limit cycle $\Gamma_{\lambda, 0}$, such that $\Gamma_{\lambda, 0} \rightarrow \Gamma$ when $\lambda \rightarrow 0$, according to Hausdorff distance.

(b) If $\Gamma$ is normally hyperbolic, then for small $\lambda, \varepsilon>0$, the regularized system (6) has a hyperbolic limit cycle $\Gamma_{\lambda, \varepsilon}$, such that $\Gamma_{\lambda, \varepsilon} \rightarrow \Gamma$, when $(\lambda, \varepsilon) \rightarrow(0,0)$, according to Hausdorff distance.

Theorem 3.1 is proved in Section 4. It is illustrated by the next example.

Example. Consider the following piecewise slow-fast system in $\mathbb{R}^{3}$

$$
\dot{x}_{1}=\left\{\begin{array}{rl}
1+200\left(x_{2}-x_{1}\right), & \text { if } \quad x_{1} \leq 0 \\
-1+200\left(x_{2}-x_{1}\right), & \text { if } \quad x_{1} \geq 0
\end{array}, \quad \dot{x}_{2}=100\left(x_{2}-x_{1}\right), \quad \varepsilon \dot{y}=y .\right.
$$

The corresponding reduced system

$$
\dot{x}_{1}=\left\{\begin{array}{rl}
1+200\left(x_{2}-x_{1}\right), & \text { if } \quad x_{1} \leq 0 \\
-1+200\left(x_{2}-x_{1}\right), & \text { if } \quad x_{1} \geq 0
\end{array}, \quad \dot{x}_{2}=100\left(x_{2}-x_{1}\right), \quad y=0\right.
$$


correspond to a class presented by Sieber and Kowalczyk in [7, p. 45] taking $\theta=-1$ and an affine change of coordinate. According the authors this class has a hyperbolic periodic orbit with sewing $\Gamma$. By Theorem 3.1 the regularized system of (9) given by

$$
\left(\dot{x}_{1}, \dot{x}_{2}\right)=\left(200\left(x_{2}-x_{1}\right)+\varphi\left(\frac{x_{1}}{\lambda}\right), 100\left(x_{2}-x_{1}\right)\right), \quad y=0
$$

has a hyperbolic limit cycle $\Gamma_{\lambda, 0}$ for small values of $\lambda>0$ (see Figure 2) and the regularized system of (8) given by

$$
\left(\dot{x}_{1}, \dot{x}_{2}, \dot{y}\right)=\left(200\left(x_{2}-x_{1}\right)+\varphi\left(\frac{x_{1}}{\lambda}\right), 100\left(x_{2}-x_{1}\right), \frac{y}{\varepsilon}\right)
$$

has a hyperbolic limit cycle $\Gamma_{\lambda, \varepsilon}$ for small values of $\lambda, \varepsilon>0$ where $\varphi$ is a transition function, see Figure 2.
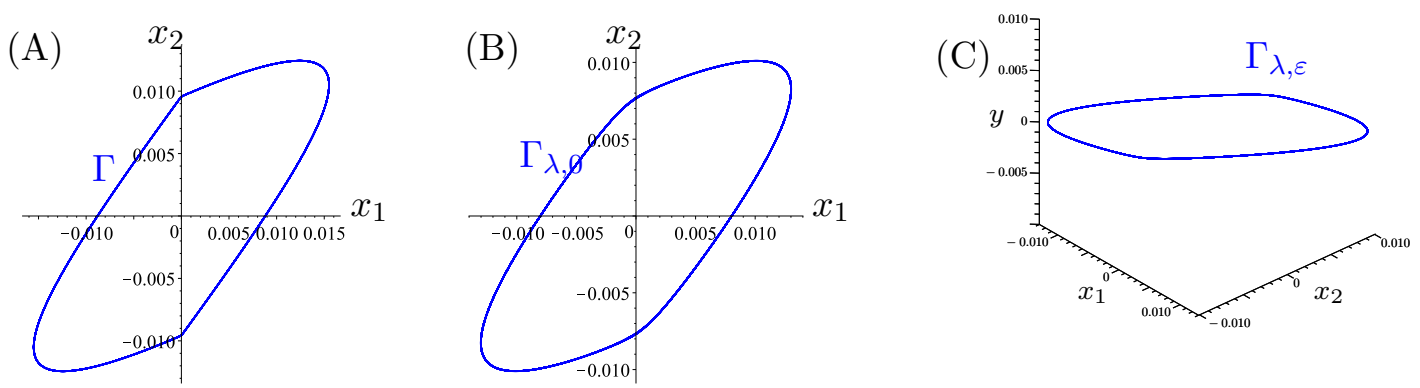

Figura 2: Figure (A) illustrates the periodic orbit with sewing $\Gamma$ of the reduced system (9). Figure (B) illustrates the limit cycle $\Gamma_{\lambda, 0}$ of the regularized system (10) with $\lambda=0.001$ and $\varphi(s)=\frac{2}{\pi} \tan ^{-1}(s / \lambda)$ and Figure (C) illustrates the limit cycle $\Gamma_{\lambda, \varepsilon}$ of system (11) with $\lambda=0.001, \varepsilon=0.01$ and $\varphi(s)=\frac{2}{\pi} \tan ^{-1}(s / \lambda)$.

\section{Proof of Theorem 3.1}

Proof of item (a). Suppose that $\Gamma$ is a periodic orbit with sewing. We can suppose without loss of generality that it is formed by two connected components $\Gamma_{0}$ and $\Gamma_{1}$. Let $p_{0}, p_{1} \in$ $\Sigma_{0} \cap \Gamma, T_{0}$ and $T_{1}$ be transversal sections to $\Gamma_{0}$ and $\Gamma_{1}$ at $p_{0}$ and $p_{1}$, respectively. Denote by $\eta_{0}: T_{0} \rightarrow T_{1}$ and $\eta_{1}: T_{1} \rightarrow T_{0}$ the half-return maps defined by the flows of $\widetilde{F}(x)$ and $\widetilde{G}(x)$, respectively. Define the return map $\eta=\eta_{1} \circ \eta_{0}$. The point $p_{0} \in T_{0}$ is the fixed point of $\eta$ which corresponds to the periodic orbit $\Gamma$. Suppose that $\Gamma$ is attractor, i.e. $\eta^{\prime}\left(p_{0}\right)<1$. The case in which $\Gamma$ is repulsive is similar. Take a orientation in $T_{0}$ and fix the origin at $p_{0}$. Let $q_{0}$ and $q_{1}$ be points of $T_{0}$ such that $q_{0}<p_{0}<q_{1}$. Thus we have that $q_{0}<\eta\left(q_{0}\right)<0$ and $0<\eta\left(q_{1}\right)<q_{1}$ because $\Gamma$ is attractor. Let $\mathcal{W}$ be a tubular neighborhood of $\Gamma$, as Figure 3 -(A) shows. Note that by construction the orbits that cross the $\operatorname{arcs} \overline{q_{0} \eta\left(q_{0}\right)}$ and $\overline{q_{1} \eta\left(q_{1}\right)}$ enter in $\mathcal{W}$. Consider small $\lambda_{0}$ such that $\eta_{\lambda}\left(q_{0}\right)>q_{0}$ and $\eta_{\lambda}\left(q_{1}\right)<q_{1}$, where $\eta_{\lambda}: T_{0} \rightarrow T_{0}$ is the return map of regularized system (7) with $0<\lambda<\lambda_{0}$. Denote by $\mathcal{W}_{\lambda}$ the region formed by orbits of system (7) by $q_{0}$ and $q_{1}$ and by $\operatorname{arcs} \overline{q_{0} \eta_{\lambda}\left(q_{0}\right)}$ and $\overline{q_{1} \eta_{\lambda}\left(q_{1}\right)}$ on $T_{0}$. By construction the orbits of system (7) enter in $\mathcal{W}_{\lambda}$. Thus the Poincaré-Bendixson Theorem 
ensures the existence of a periodic orbit $\Gamma_{\lambda, 0}$ of regularized system (7) in $\mathcal{W}_{\lambda}$ because there are no equilibrium points in $\mathcal{W}_{\lambda}$.

Now suppose that $\Gamma$ is a periodic orbit with sliding. For simplicity we suppose that $\Gamma$ has only one fold point. Let $T_{1}, T_{2}, T_{3}$ and $T_{4}$ be transversal sections to the periodic orbit $\Gamma$, as shown in Figure 3-(B). Let $p_{0} \in \Gamma \cap T_{3}$ and $q_{0}$ and $q_{1}$ be points on $T_{3}$, such that $q_{0}<p_{0}<q_{1}$. We can choose $q_{0}$ and $q_{1}$ sufficiently near to $p_{0}$ such that the orbits of $\widetilde{F}$ departing of $q_{0}$ and $q_{1}$ cross $T_{4}$ and meet the sliding region. For positive time the orbit by $q_{1}$ meets $T_{1}$ at a point $q_{2}=\left(a_{2}, b_{2}\right)$ and for negative time the orbit by $q_{1}$ meets $T_{2}$ in a point $q_{3}=\left(a_{3}, b_{3}\right)$. Take $a_{4}=\min \left\{a_{2}, a_{3}\right\}$ (in Fig. 3 -(B) we have $\left.a_{4}=a_{3}\right)$ and $a_{5}>0$ such that the orbits of $\widetilde{F}$ by $x=\left(x_{1}, x_{2}\right)$ with $x_{1}<a_{5}$ meets the sliding region. Consider the points $\left(0, a_{6}\right)$ and $\left(0, a_{7}\right)$ on the intersection of the orbit by $q_{0}$ and $\Sigma_{0}$. Consider the tubular neighborhood $\mathcal{W}$ formed by the orbit by $q_{1}$ between $T_{1}$ and $T_{2}$, by segment $\overline{\left(a_{3}, b_{2}\right)\left(a_{2}, b_{2}\right)}$ on $T_{1}$, by lines $x_{1}=a_{4}$ and $x_{1}=a_{5}$ between $T_{1}$ and $T_{2}$, by orbit by $q_{0}$ bounded by $\Sigma_{0}$ and by segments $\overline{\left(0, a_{6}\right)\left(a_{5}, b_{3}\right)}$ and $\overline{\left(0, a_{7}\right)\left(a_{5}, b_{2}\right)}$. Take $\lambda_{0}>0$ such that $-\lambda_{0} \geq a_{4}$ and $\lambda_{0} \leq a_{5}$, with $0<\lambda<\lambda_{0}$. Thus the sections $T_{3}$ and $T_{4}$ are out side of regularization zone and the flow of regularized system $(7)$ enters in $\mathcal{W}$. Again by Poincaré-Bendixson Theorem the regularized system (7) has a periodic orbit $\Gamma_{\lambda, 0}$. We can prove in details that the periodic orbit $\Gamma_{\lambda, 0}$ is hyperbolic. See for instance the references [8]. Making $\lambda \rightarrow 0$ we conclude that $\Gamma_{\lambda, 0} \rightarrow \Gamma$.
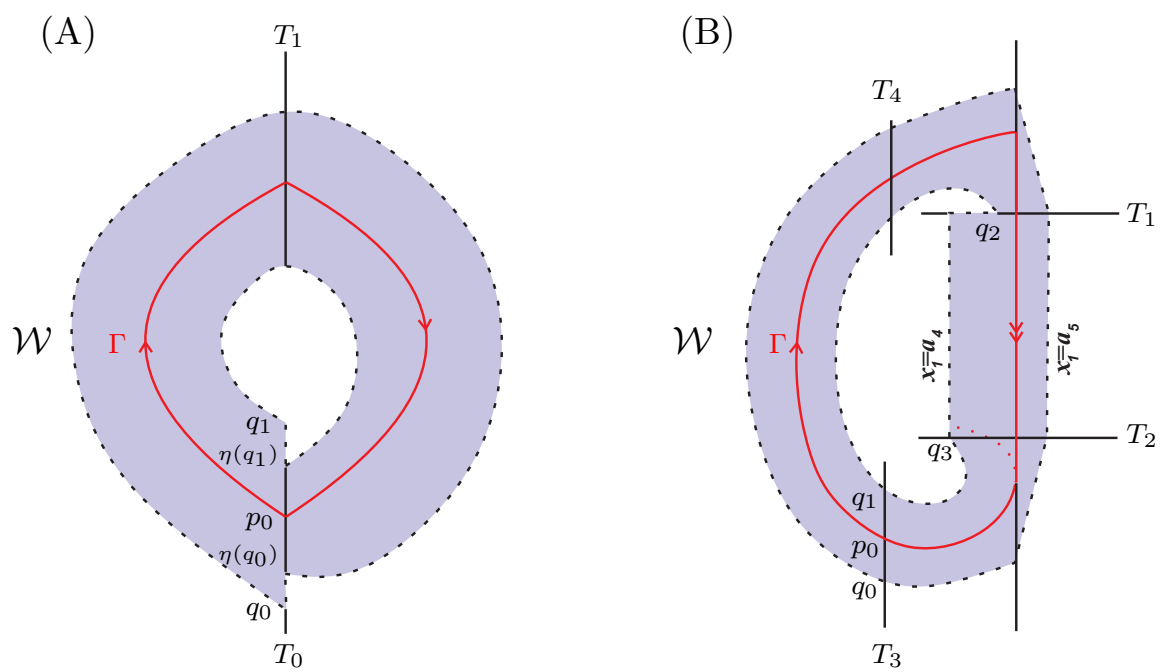

Figura 3: Figures (A) and (B) illustrate periodic orbits with sewing and with sliding and their respective tubular neighborhoods $\mathcal{W}$. For simplicity we consider $h(x, y, \varepsilon)=x_{1}$.

Proposição 4.1. If under the hypothesis of item (a) of Theorem 3.1 we add $\Gamma$ normally hyperbolic then $\Gamma_{\lambda, 0}$ is normally hyperbolic for small $\lambda>0$.

Proof. Suppose that $\Gamma$ is normally hyperbolic, i.e. $H_{y}(x, y, 0) \neq 0$ for all $(x, y) \in \Gamma$. Thus there exists a tubular neighborhood $\mathcal{V}$ of $\Gamma$ such that $H_{y}(x, y, 0) \neq 0$. If it necessary we 
can reduce the parameter $\lambda$ such that the limit cycle $\Gamma_{\lambda, 0}$ belongs to $\mathcal{V}$ and hence the result follows.

Proof of item (b). By item (a) system (7) has a hyperbolic limit cycle $\Gamma_{\lambda, 0}$ for small $\lambda>0$. Besides if $\Gamma$ is normally hyperbolic then by Proposition 4.1 the limit cycle $\Gamma_{\lambda, 0}$ is normally hyperbolic for small $\lambda>0$. As system (7) is exactly the reduced problem associated with the smooth slow-fast system (6), we can use Fenichel's Theorem ( [5]) in order to conclude that system (6) has a hyperbolic limit cycle $\Gamma_{\lambda, \varepsilon}$ for small $\lambda, \varepsilon>0$. Moreover, $\Gamma_{\lambda, \varepsilon} \rightarrow \Gamma$ when $(\lambda, \varepsilon) \rightarrow(0,0)$, according to Hausdorff distance.

\section{Acknowledgements}

The first author is supported by FAPESP grant 2013/21947-6.

\section{Referências}

[1] P.T. Cardin, P.R. da Silva and M.A. Teixeira, On singularly perturbed Filippov systems, European Journal of Applied Mathematics, vol. 24, 835-856, (2013).

[2] P.T. Cardin, P.R. da Silva and M.A. Teixeira, Geometric singular perturbation theory for non-smooth dynamical systems, Publicacions Matemàtiques, vol. Extra, 111-134, (2014).

[3] P.T. Cardin, J.R. de Moraes and P.R. da Silva, Persistence of Periodic Orbits with Sliding or Sewing by Singular Perturbation, Journal of Mathematical Analysis and Applications, vol. 423, 1166-1182, (2015).

[4] M. di Bernardo, C. J. Budd, A. R. Champneys and P. Kowalczyk, Piecewisesmooth dynamical systems - Theory and Applications, Applied Mathematical Sciences, Springer-Verlag, vol. 163, (2007).

[5] N. Fenichel, Geometric Singular Perturbation Theory for Ordinary Differential Equations, Journal of Differential Equations, vol. 31, 53-98, (1979).

[6] A. F. Filippov, Differential Equations with Discontinuous Righthand Sides, Translated from the Russian, Math. and its Applications (Soviet Series), Kluwer Academic Publishers Group, Dordrecht, vol. 18, (1988).

[7] J. Sieber and P. Kowalczyk, Small-scale Instabilities in Dynamical Systems with Sliding, Physica D. Nonlinear Phenomena, vol. 239, 44-57, (2009).

[8] J. Sotomayor and A. L. F. Machado, Structurally Stable Discontinuous Vector Fields in the Plane, Qualitative Theory of Dynamical Systems, vol. 3, 227-250, (2002).

[9] J. Sotomayor and M. A. Teixeira, Regularization of Discontinuous Vector Fields, International Conference of Differential Equations, Lisboa, 207-223, (1996). 